\title{
Preliminary Identification of Key Genes Controlling Peach Pollen Fertility Using Genome-Wide Association Study
}

\author{
Zhenyu Huang ${ }^{1,+}$, Fei Shen ${ }^{2,+}$, Yuling Chen ${ }^{1}$, Ke Cao ${ }^{1, *}$ and Lirong Wang ${ }^{1, *}$ \\ 1 Zhengzhou Fruit Research Institute, Chinese Academy of Agricultural Science, \\ Zhengzhou 450009, Henan, China; huangzhenyu@caas.cn (Z.H.); chenyuling@caas.cn (Y.C.) \\ 2 Beijing Agro-biotechnology Research Center, Beijing Academy of Agriculture and Forestry Sciences, \\ Beijing 100097, China; shenf1028@gmail.com \\ * Correspondence: wyandck@126.com (K.C.); wanglirong@caas.cn (L.W.) \\ + These authors contributed equally to this work.
}

Citation: Huang, Z.; Shen, F.; Chen, Y.; Cao, K.; Wang, L. Preliminary Identification of Key Genes Controlling Peach Pollen Fertility Using Genome-Wide Association Study. Plants 2021, 10, 242. https://doi.org/10.3390/ plants10020242

Academic Editors: Gianni Barcaccia and Alessandro Vannozzi

Received: 6 January 2021

Accepted: 25 January 2021

Published: 27 January 2021

Publisher's Note: MDPI stays neutral with regard to jurisdictional claims in published maps and institutional affiliations.

Copyright: (c) 2021 by the authors. Licensee MDPI, Basel, Switzerland. This article is an open access article distributed under the terms and conditions of the Creative Commons Attribution (CC BY) license (https:/ / creativecommons.org/licenses/by/ $4.0 /)$.

\begin{abstract}
Previous genetic mapping helped detect a $\sim 7.52 \mathrm{Mb}$ putative genomic region for the pollen fertility trait on peach Chromosome 06 (Chr.06), which was too long for candidate gene characterization. In this study, using the whole-genome re-sequencing data of 201 peach accessions, we performed a genome-wide association study to identify key genes related to peach pollen fertility trait. The significant association peak was detected at Chr.06: 2,116,368 bp, which was in accordance with the previous genetic mapping results, but displayed largely improved precision, allowing for the identification of nine candidate genes. Among these candidates, gene PpABCG26, encoding an ATP-binding cassette G (ABCG) transporter and harboring the most significantly associated SNP (Single Nucleotide Polymorphism) marker in its coding region, was hypothesized to control peach pollen fertility/sterility based on the results of gene function comparison, gene relative expression, and nucleotide sequence analysis. The obtained results will help us to understand the genetic basis of peach pollen fertility trait, and to discover applicable markers for pre-selection in peach.
\end{abstract}

Keywords: Prunus persica; pollen fertility; GWAS; candidate gene identification; ABCG transporter

\section{Background}

Peach (Prunus persica (L.) Batsch) is one of the most important fruit crops worldwide, possessing extensive genetic diversity and high economic value. Male sterility, manifesting as an abnormally developed stamen with no fertile pollen generated, is a widespread phenomenon observed in flowering plants such as peach [1]. Due to pollen abortion in peach cultivars such as 'Chinese Cling', 'Annong Shui Mi', 'Kurakato Wase', 'Wan Huang Jin', 'Da Duan Mi Lu', and 'Shenzhou Shui $\mathrm{Mi}^{\prime}$, proper pollinating trees must be planted to maintain stable fruit yields [2]. However, natural cross-pollination is easily affected by undesirable climate conditions, while manual pollination is laborious and inefficient, thus making pollen fertility a key breeding objective in peach.

Pollen fertility is closely related to the tapetum, which is the innermost layer of the anther wall, and is a secretory cell layer that functions as the source for the synthesis and transport of nutritional and structural molecules for pollen wall formation [3]. The tapetum undergoes degradation induced by programmed cell death (PCD) during meiosis, and immature or delayed tapetum PCD usually affects pollen development and male sterility $[4,5]$. In the past few years, several candidate genes reasonably associated with pollen fertility have been successfully identified in model plants, such as Arabidopsis and rice, and these genes mainly involved in three functions: (1) tapetum development (Udt1 [6], TDR [4], MS1 [7], DYT1 [8], TDF1 [9]); (2) biosynthesis and modification of various lipidic precursors for pollen wall formation in the tapetum (MS2 [10], CYP703A and CYP704B [11,12], ACOS [13], PKS [14], AMS and MS188 [15]); (3) the allocation of 
compositions from the tapetum to the anther locule for pollen wall formation (ATP-binding cassette $\mathrm{G}(A B C G)$ transporter-encoded genes [16-20]).

As for peach, even though the key factors controlling pollen fertility remain unreported, attempts have been made to discover candidate genes through forward genetic approaches. It has long been known that pollen sterility is determined by a single gene pair, $P s / p s$, with the pollen fertile allele completely dominant over the pollen sterile allele [21], and these results have been confirmed in studies on inbred $\mathrm{F}_{1}$ progeny of 'Okubo' [22], and crossbred $F_{1}$ populations of '91-42-51' $\times$ 'Ruiguang 2' [23] and 'Yumyeong' $\times$ 'Baekhyang' [24]. The Ps locus was first reported to be located between a codominant RAPD (Random Amplified Polymorphic DNA) marker, Q40cod, co-segregating with ACCAT3 (4 cM), and two co-segregating AFLP (Amplified Fragment Length Polymorphism) markers, AA-CAT4 and ACA-CAT3 $(3 \mathrm{cM}$ ), on peach linkage group (LG) 08 (corresponding to LG 06, according to the follow-up study) using a genetic map of 'Ferjalou Jalousia' $\times$ 'Fantasia' population [25]. Another RAPD marker, UBC405 2300 , has been verified as co-segregated with the Ps gene in 115 individuals of the 'Yumyeong' $\times$ 'Baekhyang' population; the recombination frequency between this marker and the Ps/ps locus is $4.3 \%$, and this marker has been found to be generally adequate to identify the $P S$ allele in other segregating progeny and commercial cultivars [24]. In the 'Ferjalou Jalousia' $\times$ 'Fantasia' $\mathrm{F}_{2}$ population, the $P_{s}$ gene is located on the top of LG 06, and the nearest marker is FG40 at $4.8 \mathrm{cM}$ [26]. Later, by screening 122 SSR (Simple Sequence Repeats) markers in 637 peach cultivars and 138 individuals of the 'Ruiguang $19^{\prime} \times$ 'Summergrand' $F_{1}$ population, two markers, CPDCT013 and CPSCT012, on the top of LG 06, were proven to be linked tightly with the $P s$ locus, being mapped at $18.9 \mathrm{cM}$ and $39.8 \mathrm{cM}$, respectively [27]. Taken together, the top region of peach LG 06 has proven to be a hotspot harboring key candidate genes underlying the pollen fertility trait.

As an alternative approach to conventional genetic mapping, the genome-wide association study (GWAS) has rapidly come into focus for the genetic dissection of complex traits by exploiting the linkage disequilibrium (LD) present among individuals from natural populations or germplasm collections, offering advantages including increased resolution, a reduced research period and improved allele number detection [28,29]. Several comprehensive GWAS projects have shed light on the genetic basis of important agronomic traits, such as fruit size and shape, fruit sugar and acid content, fruit texture, and chilling requirement, and have provided us with reliable markers for efficient genomic selection [30-36].

In the present study, we performed a large-scale GWAS using re-sequencing data generated from 201 peach accessions, aiming to identify key candidate genes controlling the pollen fertility trait, and discover applicable markers for pre-selection in peach.

\section{Results}

\subsection{Phenotyping}

By observing naturally dried anthers, a total of 180 peach accessions with abundant pollen grains were classified as fertile (Figure $1 \mathrm{a}-\mathrm{c}$ ), while 21 peach accessions with rarely observed pollen grains were classified as sterile (Figure 1d-f).

\subsection{Candidate Gene Identification via GWAS}

Approximately $378.5 \mathrm{~Gb}$ of clean sequencing reads were generated from the 201 peach accessions, with an average depth of $5.3 \times$ and an average coverage of $78.1 \%$ (Table S1). We identified a total of 1,042,687 high-quality SNPs among the accessions for the subsequent GWAS analysis.

To determine the most suitable model for peach pollen fertility trait analysis, we made a comparison between four models: (1) the general linear model without any consideration of principal component analysis (GLM-no PCA); (2) the GLM that took PCA results into account as the fixed effect (GLM-PCA); (3) the mixed linear model that incorporated kinship value (MLM-K); and (4) the MLM using the PCA results and the kinship value as a correction for population structure (MLM-K+P). As shown in Figure 2, the quantilequantile $(\mathrm{Q}-\mathrm{Q})$ plot for the GLM-no PCA model exhibited the highest deviation from the 
line of expected $P$ values versus observed ones, while the GLM-PCA model produced a distribution of $P$ values more comparable to the theoretical one; the two MLM models were better than the two GLM models in this regard but exhibited excessive correction. As such, the GLM-PCA model was adopted in our study.

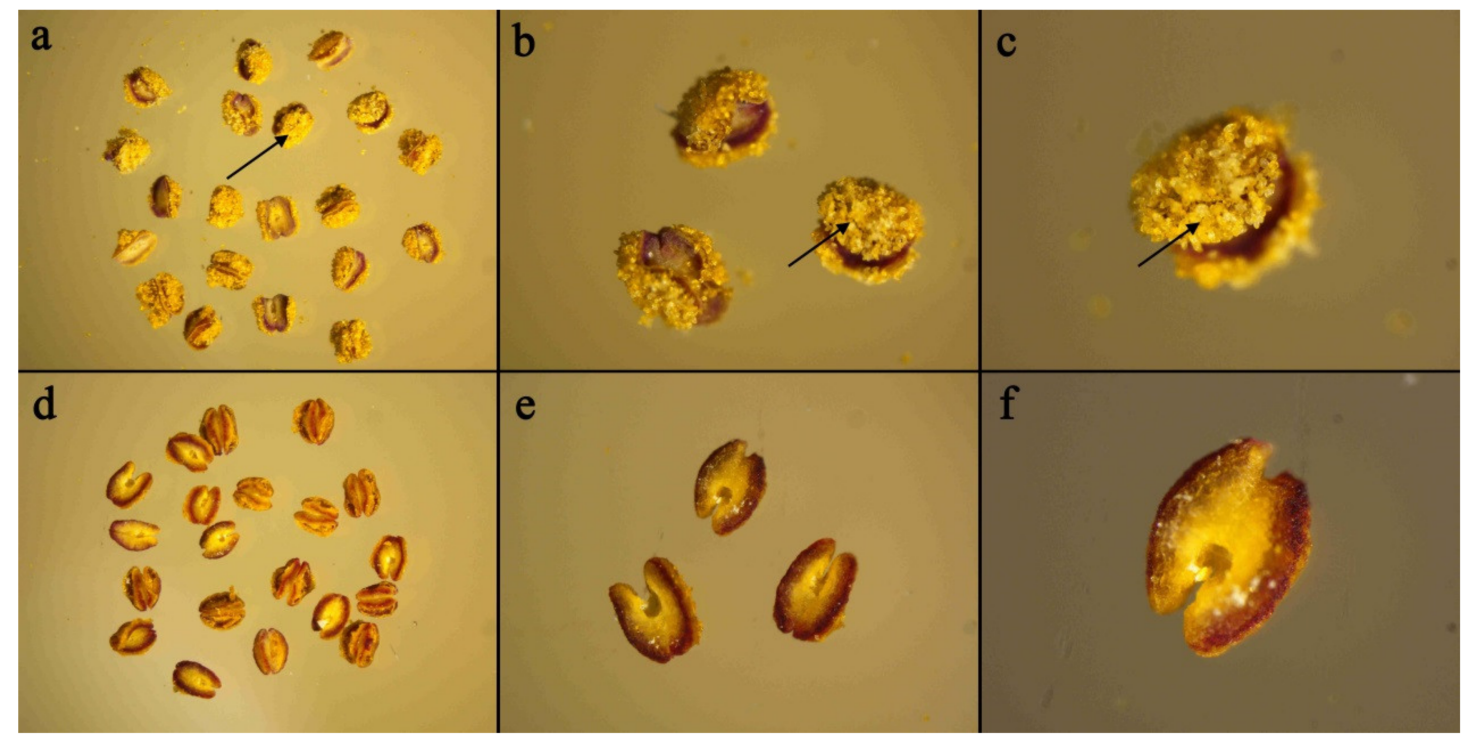

Figure 1. The anther observation of the peach fertile/sterile phenotype under different magnifications. The black arrows indicate the mature pollen grains. $(\mathbf{a}-\mathbf{c})$ indicate the fertile phenotype with abundant pollen grains in the naturally dried anthers. (d-f) indicate the sterile phenotype with no pollen grains observed.
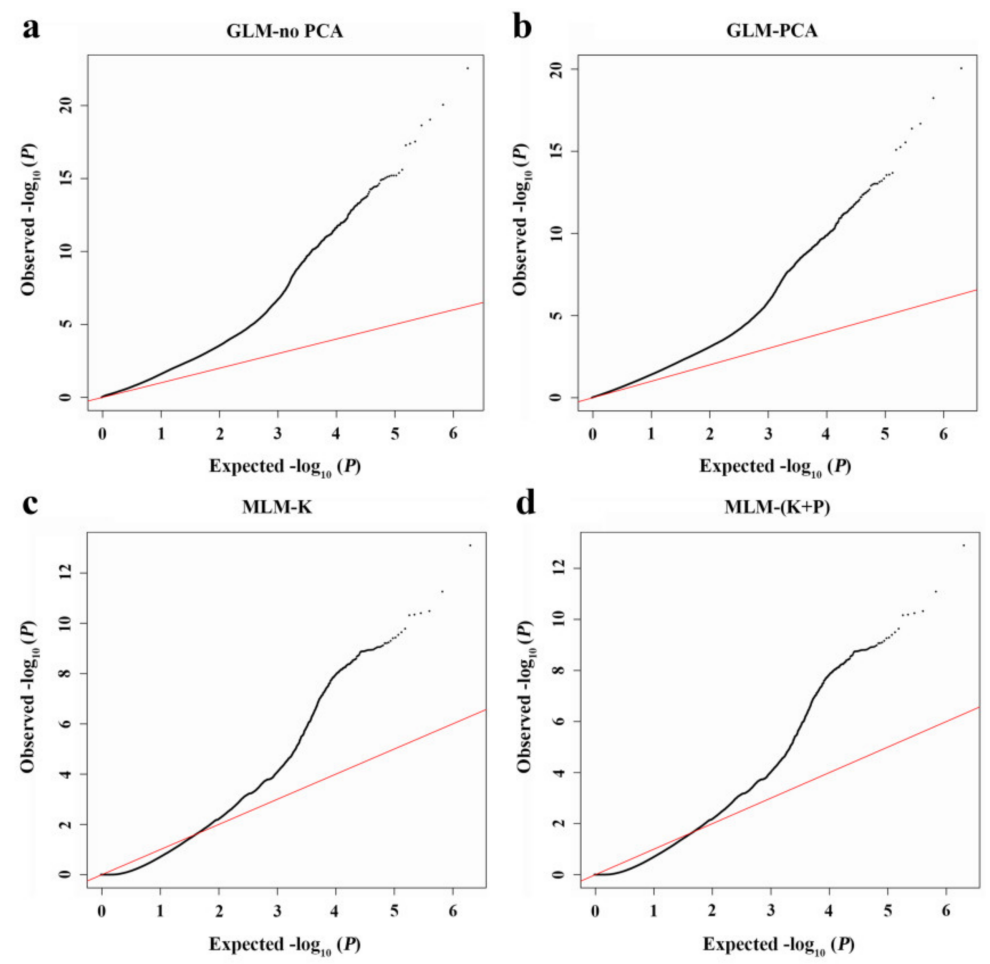

Figure 2. Quantile-quantile (Q-Q) plot of the four analysis models used in the genome-wide association study (GWAS) for peach pollen fertility trait. The red line indicates the expected line under the null distribution. The black line represents the $P$ values observed using the (a) GLM-no PCA, (b) GLM-PCA, (c) MLM-K, and (d) MLM-(K+P) models, respectively. 
The additional association analysis helped us determine the reasonable loci related to peach pollen fertility (Figure 3). As revealed by the Q-Q plot and Manhattan plots, a significant association peak was located on Chr.06: 2,116,368 bp, with a $-\log _{10}(P)$ of 19.8 . Considering that the LD decay was about $20-50 \mathrm{~kb}$ for the different subgroups of cultivated peach [30], we subsequently performed candidate gene screening in the region that covered $\pm 25 \mathrm{~kb}$ on either side of the significant association peak. This allowed us to identify nine candidate genes potentially responsible for the peach pollen fertility trait (Table 1).

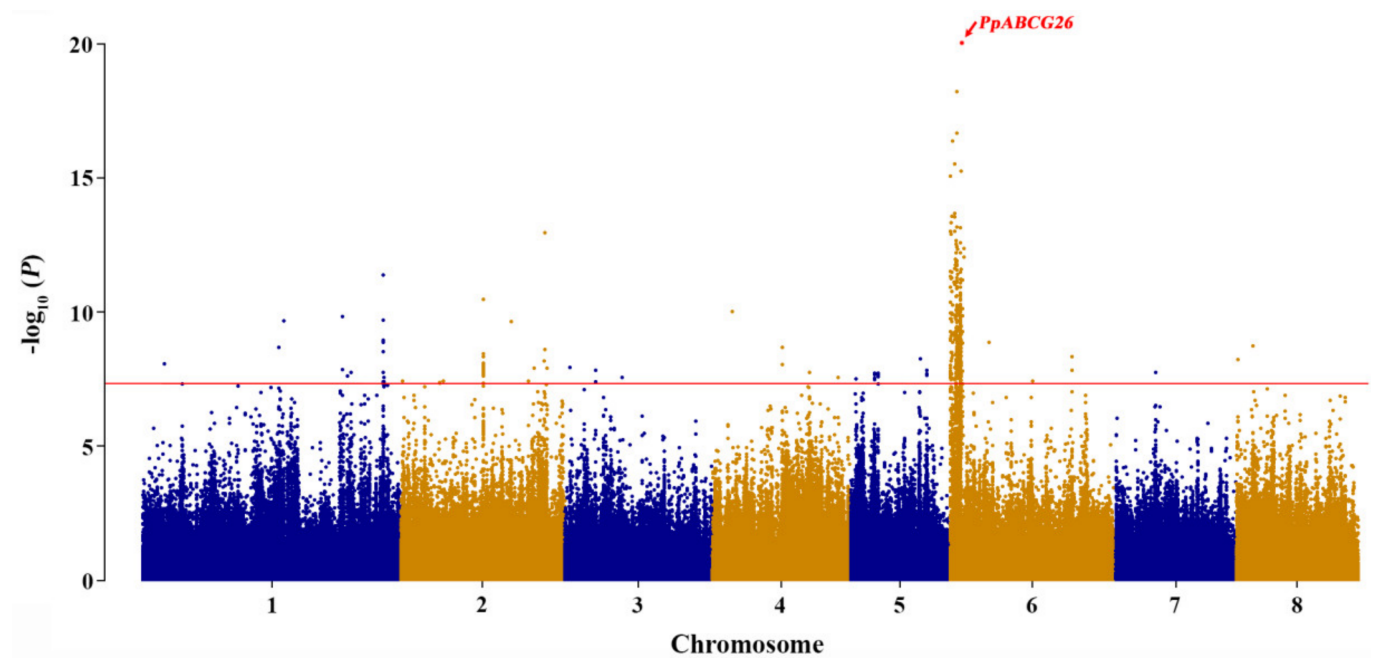

Figure 3. Genome-wide association study result of peach pollen fertility trait. The $y$-axis indicates the association value of the SNPs, and the $x$-axis indicates their positions on the chromosomes. The horizontal red line represents the significance threshold (7.32) for the association of peach pollen fertility trait.

Table 1. Information pertaining to candidate genes for peach pollen fertility.

\begin{tabular}{|c|c|c|}
\hline Gene ID & Physical Position (bp) & Functional Annotation \\
\hline Prupe.6G026600 & Chr.06: 2,092,736-2,096,757 & Heterogeneous nuclear ribonucleoprotein 1 \\
\hline Prupe.6G026700 & Chr.06: $2,100,008-2,106,576$ & Kinase-related protein of unknown function \\
\hline Prupe.6G026800 & Chr.06: $2,106,907-2,110,035$ & Nucleotide/sugar transporter family protein \\
\hline Prupe.6G026900 & Chr.06: 2,111,266-2,114,599 & Coiled-coil domain-containing protein 22 \\
\hline Prupe.6G027000 & Chr.06: $2,115,131-2,118,488$ & ABC transporter G family member 26 \\
\hline Prupe.6G027100 & Chr.06: 2,118,854-2,122,002 & Transmembrane protein 245 \\
\hline Prupe.6G027200 & Chr.06: 2,122,159-2,125,309 & Protein CWC15 homolog A \\
\hline Prupe.6G027300 & Chr.06: 2,132,488-2,134,587 & Viral IAP-associated factor homolog \\
\hline Prupe.6G027400 & Chr.06: 2,135,267-2,145,546 & $\begin{array}{c}\text { Mediator of RNA polymerase II transcription } \\
\text { subunit } 15 a\end{array}$ \\
\hline
\end{tabular}

Note: Bp, basepair. Chr, chromosome.

\subsection{Candidate Gene Expression Analysis}

We measured the expression profiles of the nine candidate genes related to peach pollen fertility in the anther samples from the fertile cultivar 'Da Hong Pao' and the sterile cultivar 'Annong Shui Mi' (Figure 4). Except for the differentially expressed gene Prupe.6G027000, the other eight candidate genes exhibited either no expression in peach anther or undifferentiated expression between fertile and sterile peach anthers. Combined with the functional annotation of gene Prupe.6G027000 as an ABC transporter G family member 26-the homologs of which have been proven to play a crucial role in pollen grain development in rice and Arabidopsis, as mentioned above-here we took said gene as a strong candidate in peach fertility determination and designated it as PpABCG26. 


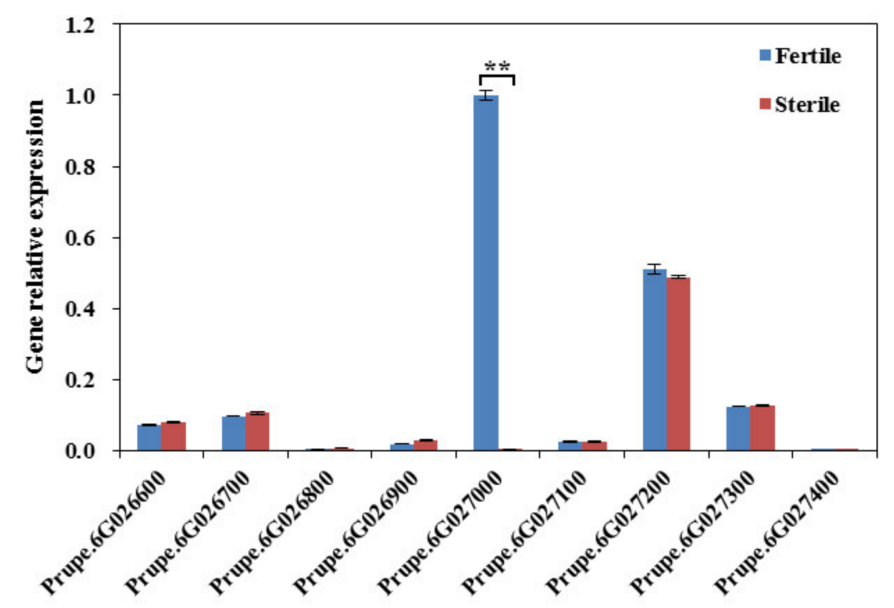

Figure 4. Expression patterns of the nine candidate genes related to peach pollen fertility. The blue bars and red bars indicate the relative expression in the anther samples of the fertile cultivar ' $\mathrm{Da}$ Hong Pao' and sterile cultivar 'Annong Shui Mi', respectively. ${ }^{* *} p<0.01$.

\subsection{Candidate Gene Sequence Analysis}

The amino acid sequences of the ABCG26 proteins of Arabidopsis (Arabidopsis thaliana), peach (Prunus persica), apricot (Prunus armeniaca), Chinese plum (Prunus salicina), sweet cherry (Prunus avium), apple (Malus domestica), and rice (Oryza sativa) were retrieved from The Arabidopsis Information Resource database (TAIR; https://www.arabidopsis.org/), the Genome Database of Rosaceae (GDR; https:/ / www.rosaceae.org/), and the China Rice Data Center (http:/ / www.ricedata.cn/), and were then subjected to sequence alignment and phylogenetic analysis, resulting in a sequence identity of $81.88 \%$ across different species (Figure 5, Figure S1). As revealed by the GWAS results, the significant association peak was at Chr.06: 2,116,368 bp, which displayed polymorphism (T/C) among the 201 peach accessions and was precisely located in the coding sequence (+824 bp) of PpABCG26. We then validated this SNP (T/C) using Sanger sequencing in ten randomly selected peach cultivars (five fertile and five sterile). As shown in Figure 6a, the T genotype and C genotype were tightly associated with fertile and sterile phenotypes, respectively. Amino acid translation revealed a transition from Leucine (codon CTT) to Proline (codon CCT). Additionally, using TMHMM Server v. 2.0 (http:/ / www.cbs.dtu.dk/services/TMHMM/), we performed a prediction of transmembrane helices in the PpABCG26 protein (Figure 6b) and found that the resultant amino acid change from $\mathrm{Leu}_{275}$ to Pro was predicted to be located on the intracellular binding domain instead of the six transmembrane domains. This suggests that the nonsynonymous SNP (T/C) possibly affected the binding between the transporter and pollen grain development-related substrates and finally led to pollen sterility in peach.

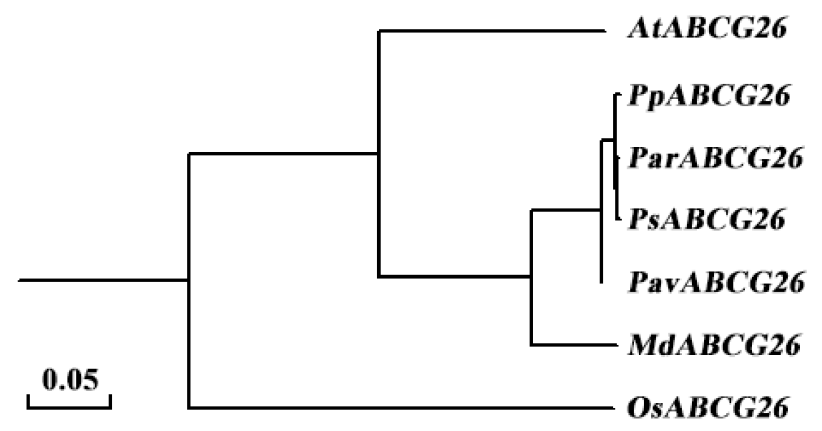

Figure 5. Phylogenetic tree of the ABCG26 proteins of different plant species. The species, from top to bottom, are Arabidopsis (Arabidopsis thaliana), peach (Prunus persica), apricot (Prunus armeniaca), Chinese plum (Prunus salicina), sweet cherry (Prunus avium), apple (Malus domestica), and rice (Oryza sativa). 
a

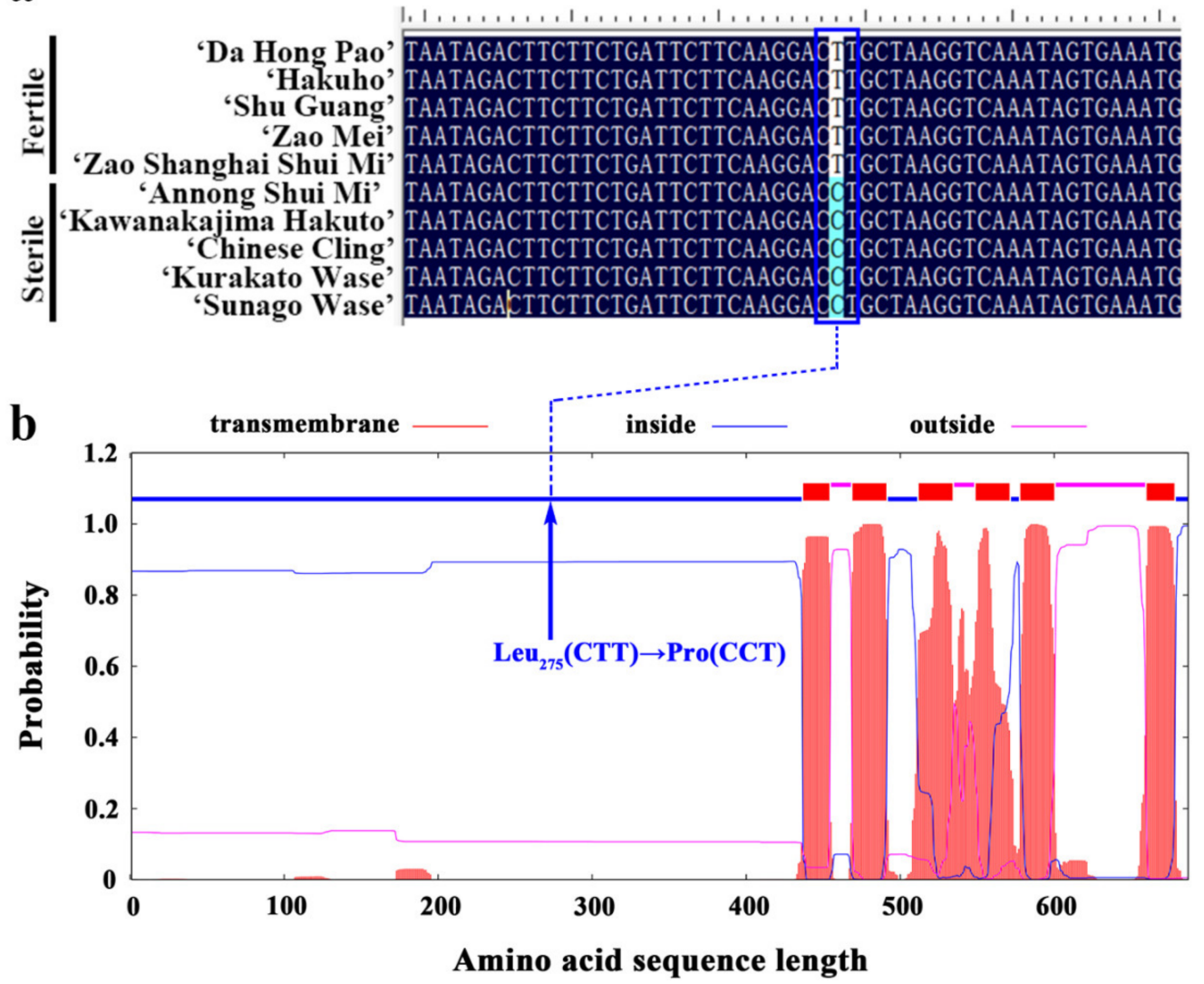

Figure 6. Sequence analysis of the candidate gene $P p A B C G 26$. (a) The sequence alignment of $P p A B C G 26$ in the five fertile and five sterile peach cultivars. The blue box indicates the nonsynonymous SNP $(\mathrm{T} / \mathrm{C})$ at +824 bp in the coding region. (b) The predicted transmembrane helices of PpABCG26. The blue arrow indicates the resultant amino acid change from $\mathrm{Leu}_{275}$ (CTT) to Pro (CCT).

\section{Discussion}

Discovering the genetic factors underlying important agronomic traits continues to draw breeders' attention since it could improve genomic selection efficiency by providing valuable markers. In the past few decades, for the peach pollen fertility/sterility trait, the top region of $\mathrm{Chr} .06(0-7.52 \mathrm{Mb})$ was characterized as a hotspot by using genetic mapping in different hybrid populations, but it remained difficult to focus on specific genes as the SSR markers used were employed at a low density [24-27]. With the capabilities of sequencing technologies, high-density molecular markers have been widely adopted in genetic basis dissection [30-36]. In our study, using the high-quality SNPs generated from the re-sequencing data of 201 peach accessions, a significant association peak was detected at Chr.06: 2,116,368 bp (Figure 3), which is in accordance with the previous genetic mapping results but displays largely improved precision, allowing follow-up candidate gene identification (Table 1).

Combining the results of GWAS, functional annotation, sequence alignment, gene relative expression analysis, and SNP validation, the gene PpABCG26 was speculated to be responsible for pollen fertility/sterility in peach (Figure 3,4 and 6). Through evolution, ABCGs have emerged as one of the most conserved but also divergent proteins that safeguard the formation of the male gametophyte via mediating the lipid metabolism and, in particular, the transport of the lipidic and phenolic precursors across both sides of the anther layers, to form the two most important protective barriers of pollen development [38]. By performing sequence alignment and phylogenetic analysis, we found a sequence identity of $81.88 \%$ across different flowering species belonging to both Monocotyledoneae and Dicotyledoneae (Figure 5, Figure S1). As a membrane protein, the critical role of ABCG26 in exine formation and pollen development has been well studied in Arabidopsis 
and rice and is consistent with the model by which ABCG26 transports sporopollenin precursors across the tapetum plasma membrane into the locule for polymerization on developing microspore walls, both of which are plant-specific structures critical to pollen development [16-20].

By genotyping the gene-contained SNP (T/C) in five fertile and five sterile peach cultivars, the $\mathrm{T}$ genotype and the $\mathrm{C}$ genotype were shown to be tightly associated with the fertile and sterile phenotypes, respectively (Figure 6a). However, by analyzing the re-sequencing data of the 201 peach accessions, we noticed that, for the 21 sterile accessions, 9 of them displayed the heterozygous $\mathrm{T} / \mathrm{C}$ or homozygous $\mathrm{T} / \mathrm{T}$ genotype, which was inconsistent with the theological homozygous $\mathrm{C} / \mathrm{C}$ genotype for sterile cultivars; as regards the 180 fertile accessions, all of them displayed the heterozygous T/C or homozygous $\mathrm{T} / \mathrm{T}$ genotype, which was in accordance with the theological ones (Table S1). It would be reasonable to assume that, besides the ABCG26 transporter, some other factors that work collaboratively to transport multiple precursors for cutin, wax, sporopollenin, tryphine, intine, and nexine formation may lead to a similar sterile phenotype. In Arabidopsis, tapetal cells were shown to generate precursors for the formation of sexine, tryphine, and nexine, which are transferred to other locules by AtABCG26, AtABCG9 and AtABCG31, and AtABCG1 and AtABCG16, crossing the tapetal cells' plasma membrane, then be transferred to the microspores surface by unknown transporters. AtABCG1 and AtABCG16 also export intine precursors across microspores' plasma membranes for intine development. In rice, both OsABCG26 and OsABCG15 collaboratively regulate the transport of anther cuticle and sporopollenin precursors; while OsABCG26 mainly transports wax and cutin precursors toward the anther surface for anther cuticle formation, OsABCG15 transport sporopollenin precursors from the tapetum to the anther locule for exine formation $[18,37,38]$. The natural variation in the coding sequence $(+824 \mathrm{bp})$ of gene PpABCG26 was a sufficient, but not necessary condition for the peach pollen fertile/sterile phenotype.

\section{Materials and Methods}

\subsection{Plant Materials}

In total, 201 peach accessions were used for whole-genome re-sequencing and subsequent GWAS analysis (Table S1). The fertile cultivar 'Da Hong Pao' and the sterile cultivar 'Annong Shui Mi' were selected for gene nucleotide sequence analysis and gene relative expression analysis. All the peach accessions were planted at the National Germplasm Repository of Peach in Zhengzhou, Henan, China $\left(35^{\circ} 09^{\prime} \mathrm{N}, 113^{\circ} 47^{\prime} \mathrm{E}\right)$; these trees were trained to the " $\mathrm{Y}^{\prime}$ " system at a density of $2.0 \times 5.0 \mathrm{~m}$ and were maintained under identical management operations.

\subsection{Phenotyping}

We determined the fertility/sterility of the peach accessions by observing the presence of pollen grains in the naturally dried anthers. The fresh anthers were collected, stored under cool and dry conditions for $72 \mathrm{~h}$, and then directly observed using a microscope [39]. Accessions with abundant pollen grains were classified as fertile (Figure 1a-c), while accessions with rarely observed pollen grains were classified as sterile (Figure $1 \mathrm{~d}-\mathrm{f}$ ).

\subsection{Nucleic Acid Isolation}

Genomic DNA was extracted using a QIAGEN ${ }^{\circledR}$ Genomic kit (Qiagen Co., Ltd., Hilden, Germany) from young leaves bursting after blooming, and the total RNA of anther samples was isolated using a Plant Total RNA Extraction Kit (Huayueyang Bio Technology Co., Ltd., Beijing, China), deferring to the manufacturer's instructions. The quality and quantity of the isolated DNA and RNA were separately checked via electrophoresis on a $0.75 \%$ agarose gel and a NanoDrop ${ }^{\mathrm{TM}} \mathrm{D}-1000$ spectrophotometer (NanoDrop Technologies, Wilmington, DE, USA). 


\subsection{DNA Sequencing and Association Study}

The whole-genome re-sequencing of the 201 peach accessions was completed in our previous work [34]. The library insert size was $500 \mathrm{bp}$, and the pair-end read size was $150 \mathrm{bp}$. All libraries were sequenced using the Illumina Hiseq ${ }^{\mathrm{TM}} \mathrm{X}$-Ten platform (Illumina, San Diego, CA, USA) by Annoroad Gene Technology (Beijing, China). The qualified pairedend reads of each accession were aligned against the peach reference genome v2.0 [40] using BWA v0.7.12-r1039 [41], and SNPs were identified using the Genome Analysis Toolkit (GATK) [42]. The SNPs with missing data $\geq 20 \%$ and minor allele frequencies (MAF) $<2 \%$ in the population were discarded, leaving 1,042,687 high-quality SNPs for the following GWAS analysis.

Based on the re-sequencing data of 201 peach accessions, we compared four models with TASSEL v4.1 to determine the best one for our association analysis [43]: the GLM-no PCA, the GLM-PCA, the MLM- $k$, and the MLM-K+P models. The specific commands to run each model were as follows: (1) GLM-no PCA: ./tassel4.0_standalone/run_pipeline.pl -fork1 -h./genotype.txt -fork2 -r./phenotype.txt -combine4 -input1 -input2 -intersect -glm export glm_output -runfork1 -runfork2; (2) GLM-PCA: ./tassel4.0_standalone/run_pipeline. $\mathrm{pl}$-fork1 -h./genotype.txt -fork2 -r./phenotype.txt -fork3 -q./PCA.cov -combine5 -input1 -input2 -input3 -intersect -glm -export glm_output -runfork1 -runfork2 -runfork3; (3) MLM$\mathrm{K}:$./tassel4.0_standalone/run_pipeline.pl -fork1 -h./genotype.txt -fork2 -r./phenotype.txt -fork3 -k./kinship.txt -combine4 -input1 -input2 -intersect -combine5 -input3 -input4 $\mathrm{mlm}$-mlmVarCompEst P3D -mlm CompressionLevel Optimum -export mlm_output runfork1 -runfork2 -runfork3; and (4) MLM-K+P: ./tassel4.0_standalone/run_pipeline.pl -fork1 -h./genotype.txt -fork2 -r. / phenotype.txt -fork3 -q./PCA.cov -fork4 -k./ kinship.txt -combine5 -input1 -input2 -input3 -intersect -combine6 -input5 -input4 -mlm -mlmVarCompEst P3D -mlmCompressionLevel None -export mlm_output -runfork1 -runfork2 -runfork3 -runfork4 [32].

A stringent Bonferroni correction was used to screen obvious association signals based on the $P$ value, calculated by dividing 0.05 by the number of SNPs. Manhattan and quantilequantile $(\mathrm{Q}-\mathrm{Q})$ plots were generated in $\mathrm{R}$ v3.5.1 using the package qqman v0.1.2 [44].

\subsection{Candidate Gene Sequence Analysis}

The reference nucleotide sequences of candidate genes were obtained from the Genome Database of Rosaceae (GDR; https://www.rosaceae.org/). The candidate gene-specific primers (Sense: 5' CATCAAAGGCATATCAGG 3'; Anti-sense: 5' TTGTCCAGTGGCTAAATC $\left.3^{\prime}\right)$ were designed using the Primer-BLAST tool integrated with the National Center for Biotechnology Information Database (NCBI) (https:/ / www.ncbi.nlm.nih.gov/tools/primerblast/). The PCRs were performed via procedures set according to the product lengths and primer annealing temperatures. The PCR products comprising SNP loci of interest were then subjected to Sanger sequencing at Sangon Biotech (Shanghai, China) Co., Ltd.

\subsection{Candidate Gene Expression Analysis}

The fresh anther samples of the fertile cultivar 'Da Hong Pao' and the sterile cultivar 'Annong Shui Mi' were collected every three days from 1 March to 15 April in 2019, corresponding with the period from the sporogenous cell formation stage to the balloon-flower stage [45]. The anther samples of the two cultivars were mixed respectively and then subjected to RNA isolation. First-strand cDNA synthesis was carried out using $1.0 \mathrm{mg}$ qualified RNA and the Transcriptor First Strand cDNA synthesis kit (Takara, Dalian, China) according to the manufacturer's instructions. The gene-specific primers were also designed using the Primer-BLAST tool (Table S2). The qRT-PCRs were performed using the LightCycler System (Roche LightCycler 480, Roche Diagnostics, Basel, Switzerland) following the manufacturer's instructions. The gene relative expression levels were calculated with the $2^{-\triangle \Delta C T}$ method. 


\section{Conclusions}

By conducting GWAS on 201 peach accessions, we detected a significant association peak for the peach pollen fertility trait at Chr.06: 2,116,368 bp, which was located in the coding region of the gene $P p A B C G 26$. Based on the results of the gene function comparison, gene relative expression, and nucleotide sequence analysis, $P p A B C G 26$ was proposed as a key factor in controlling peach pollen fertility/sterility. These results will help us to understand the genetic basis of the peach pollen fertility trait and to discover applicable markers for pre-selection in peach.

Supplementary Materials: Figure S1. Sequence alignments of ABCG26 proteins of Arabidopsis (Arabidopsis thaliana), peach (Prunus persica), apricot (Prunus armeniaca), Chinese plum (Prunus salicina), sweet cherry (Prunus avium), apple (Malus domestica), and rice (Oryza sativa). Table S1. Sequencing information of the 201 peach accessions used for GWAS analysis. Table S2. Gene-specific primers for qRT-PCR.

Author Contributions: Conceptualization, L.W., K.C., and Y.C.; methodology, Z.H. and F.S.; investigation, Z.H.; data curation, Z.H., F.S., and K.C.; formal analysis, Z.H., F.S., and K.C.; writing-original draft preparation, Z.H.; writing—review and editing, Z.H. and F.S.; supervision, L.W., K.C. and Y.C. All authors have read and agreed to the published version of the manuscript.

Funding: This work was supported by the National Key Research and Development Program (2019YFD1000203) and the Agricultural Science and Technology Innovation Program (CAAS-ASTIP2016-ZFRI-01).

Institutional Review Board Statement: Not applicable.

Informed Consent Statement: Not applicable.

Data Availability Statement: The referenced SNPs have also been deposited in the Figshare database (https:/ / figshare.com/s/7e177dad8e036742c0fb).

Conflicts of Interest: The authors declare that they have no competing interest.

\section{References}

1. Kaul, M.L.H. Male Sterility in Higher Plants; Springer: New York, NY, USA, 1988; pp. 2-5.

2. Wang, L.; Zhu, G.; Fang, W.; Cao, K.; Wang, X.; Chen, C.; Zhao, P.; Wang, X. Peach Genetic Resources in China; China Agricultural Press: Beijing, China, 2012; pp. 118-119.

3. Goldberg, R.B.; Beals, T.P.; Sanders, P.M.; Goldberg, B.; Beals, T.P.; Sanders, P.M. Anther Development: Basic Principles and Practical Applications. Plant Cell 2015, 5, 1217-1229.

4. Li, N.; Liu, H.; Yin, C.; Li, X.; Liang, W.; Yuan, Z.; Xu, B.; Chu, H.; Wang, J.; Wen, T.; et al. The Rice Tapetum Degeneration Retardation Gene Is Required for Tapetum Degradation and Anther Development. Plant Cell 2006, 18, 2999-3014. [CrossRef]

5. Xu, J.; Yang, C.; Yuan, Z.; Zhang, D.; Gondwe, M.Y.; Ding, Z.; Liang, W.; Zhang, D.; Wilson, Z.A. The ABORTED MICROSPORES Regulatory Network Is Required for Postmeiotic Male Reproductive Development in Arabidopsis thaliana. Plant Cell 2010, 22, 91-107. [CrossRef]

6. Jung, K.; Han, M.; Lee, Y.; Kim, Y.; Hwang, I.; Kim, M.; Kim, Y.; Nahm, B.H.; An, G. Rice Undeveloped Tapetum1 Is a Major Regulator of Early Tapetum Development. Plant Cell 2005, 17, 2705-2722. [CrossRef]

7. Vizcay-barrena, G.; Wilson, Z.A. Altered tapetal PCD and pollen wall development in the Arabidopsis ms1 mutant. J. Exp. Bot. 2006, 57, 2709-2717. [CrossRef]

8. Zhang, W.; Sun, Y.; Timofejeva, L.; Chen, C.; Grossniklaus, U.; Ma, H.; Cells, S.; Exs, E.M.S. Regulation of Arabidopsis tapetum development and function by DYSFUNCTIONAL TAPETUM1 (DYT1) encoding a putative bHLH transcription factor. Development 2006, 3095, 3085-3095. [CrossRef]

9. Zhu, J.; Chen, H.; Li, H.; Gao, J.; Jiang, H.; Wang, C.; Guan, Y.; Yang, Z. Defective in Tapetal Development and Function 1 is essential for anther development and tapetal function for microspore maturation in Arabidopsis. Plant Cell 2008, 55, 266-277. [CrossRef] [PubMed]

10. Wang, A.; Xia, Q.; Xie, W.; Dumonceaux, T.; Zou, J.; Datla, R.; Selvaraj, G. Male gametophyte development in bread wheat (Triticum aestivum L.): Molecular, cellular, and biochemical analyses of a sporophytic contribution to pollen wall ontogeny. Plant J. 2002, 30, 613-623. [CrossRef] [PubMed]

11. Morant, M.; Schaller, H.; Pinot, F. CYP703 Is an Ancient Cytochrome P450 in Land Plants Catalyzing in-Chain Hydroxylation of Lauric Acid to Provide Building Blocks for Sporopollenin Synthesis in Pollen. Plant Cell 2007, 19, 1473-1487. [CrossRef] [PubMed] 
12. Dobritsa, A.A.; Shrestha, J.; Morant, M.; Pinot, F.; Matsuno, M.; Swanson, R.; Møller, B.L.; Preuss, D.; Frederiksberg, C.; Denmark, M.M.; et al. CYP704B1 Is a Long-Chain Fatty Acid v -Hydroxylase Essential for Sporopollenin Synthesis in Pollen. Plant Physiol. 2009, 151, 574-589. [CrossRef]

13. Yang, X.; Liang, W.; Chen, M.; Zhang, D.; Zhao, X. Rice fatty acyl-CoA synthetase OsACOS12 is required for tapetum programmed cell death and male fertility. Planta 2017, 246, 105-122. [CrossRef] [PubMed]

14. Zou, T.; Liu, M.; Xiao, Q.; Wang, T.; Chen, D.; Luo, T.; Yuan, G.; Li, Q.; Zhu, J.; Liang, Y.; et al. OsPKS2 is required for rice male fertility by participating in pollen wall formation. Plant Cell Rep. 2018, 37, 759-773. [CrossRef]

15. Wang, K.; Guo, Z.; Zhou, W.; Zhang, C.; Zhang, Z.; Lou, Y.; Xiong, S.; Yao, X.; Fan, J.; Zhu, J.; et al. The Regulation of Sporopollenin Biosynthesis Genes for Rapid Pollen Wall Formation. Plant Physiol. 2018, 178, 283-294. [CrossRef]

16. Quilichini, T.D.; Friedmann, M.C.; Samuels, A.L.; Douglas, C.J. ATP-Binding Cassette Transporter G26 Is Required for Male Fertility and Pollen Exine Formation. Plant Physiol. 2010, 154, 678-690. [CrossRef] [PubMed]

17. Kuromori, T.; Ito, T.; Sugimoto, E.; Shinozaki, K. Arabidopsis mutant of AtABCG26, an ABC transporter gene, is defective in pollen maturation. J. Plant Physiol. 2011, 168, 2001-2005. [CrossRef] [PubMed]

18. Choi, H.; Ohyama, K.; Kim, Y.; Jin, J.; Lee, S.B.; Yamaoka, Y.; Muranaka, T.; Suh, M.C.; Fujioka, S.; Lee, Y. The Role of Arabidopsis ABCG9 and ABCG31 ATP Binding Cassette Transporters in Pollen Fitness and the Deposition of Steryl Glycosides on the Pollen Coat. Plant Cell 2014, 26, 310-324. [CrossRef] [PubMed]

19. Wu, L.; Guan, Y.; Wu, Z.; Yang, K.; Lv, J.; Converse, R. OsABCG15 encodes a membrane protein that plays an important role in anther cuticle and pollen exine formation in rice. Plant Cell Rep. 2014, 33, 1881-1899. [CrossRef]

20. Chang, Z.; Chen, Z.; Yan, W.; Xie, G.; Lu, J.; Wang, N.; Lu, Q.; Yao, N.; Yang, G.; Xia, J.; et al. An ABC transporter, OsABCG26, is required for anther cuticle and pollen exine formation and pollen-pistil interactions in rice. Plant Sci. 2016, 253, 21-30. [CrossRef]

21. Scott, D.H.; Weinberger, J.H. Inheritance of pollen sterility in some peach varieties. Proc. Am. Soc. Hortic. Sci. 1944, 9, 187-188.

22. Zhang, L.; Xiao, X.; Wang, X.; Dai, G. Variability and Inheritance of Some Characters in Self-crossed Progeny of Okubo Peach Cultivar. J. Fruit Sci. 2004, 21, 308-310.

23. Yu, M.; Ma, R.; Shen, Z.; Zhang, Z. Molecular Markers Linked to Specific Characteristics of Prunus Persica (L.) Bastch. Jiangsu Acta Hortic. Sin. 2004, 33, 511-517.

24. Jun, J.H.; Chung, K.H.; Jeong, S.B.; Lee, H.J. An RAPD marker linked to the pollen sterility gene ps in peach (Prunus persica). J. Hortic. Sci. Biotechnol. 2004, 79, 587-590. [CrossRef]

25. Dirlewanger, E.; Pronier, V.; Parvery, C.; Rothan, C.; Guye, A.; Monet, R. Genetic linkage map of peach [Prunus persica (L.) Batsch] using morphological and molecular markers. Theor. Appl. Genet. 1998, 97, 888-895. [CrossRef]

26. Dirlewanger, E.; Cosson, P.; Boudehri, K.; Renaud, C.; Capdeville, G.; Tauzin, Y.; Laigret, F.; Moing, A. Development of a second-generation genetic linkage map for peach [Prunus persica (L.) Batsch] and characterization of morphological traits affecting flower and fruit. Tree Genet. Genomes 2006, 3, 1-13. [CrossRef]

27. Cao, K.; Wang, S.; Zhu, G.; Fang, W.; Chen, C.; Wang, L. Study on Relationship Between Pollen Fertility and Anther Color and Its SSR Marker Screening in Peach. J. Plant Genet. Resour. 2010, 11, 817-822.

28. Hall, D.; Tegström, C.; Ingvarsson, P.K. Using association mapping to dissect the genetic basis of complex traits in plants. Brief. Funct. Genom. Proteom. 2010, 9, 157-165. [CrossRef]

29. Chen, Y. Dissection of Agronomic Traits in Crops by Association Mapping. In Diagnostics in Plant Breeding; Lübberstedt, T., Varshney, R., Eds.; Springer: Dordrecht, The Netherlands, 2013; pp. 119-142.

30. Cao, K.; Zheng, Z.; Wang, L.; Liu, X.; Zhu, G.; Fang, W.; Cheng, S.; Zeng, P.; Chen, C.; Wang, X.; et al. Comparative population genomics reveals the domestication history of the peach, Prunus persica, and human influences on perennial fruit crops. Genome Biol. 2014, 15, 1-15. [CrossRef]

31. Micheletti, D.; Dettori, M.T.; Micali, S.; Aramini, V.; Pacheco, I.; da Silva Linge, C.; Foschi, S.; Banchi, E.; Barreneche, T.; Quilot-Turion, B.; et al. Whole-genome analysis of diversity and SNP-major gene association in peach germplasm. PLoS ONE 2015, 10, 1-19. [CrossRef]

32. Cao, K.; Zhou, Z.; Wang, Q.; Guo, J.; Zhao, P.; Zhu, G.; Fang, W.; Chen, C.; Wang, X.; Wang, X.; et al. Genome-wide association study of 12 agronomic traits in peach. Nat. Commun. 2016, 7, 1-10. [CrossRef]

33. Yu, Y.; Fu, J.; Xu, Y.; Zhang, J.; Ren, F.; Zhao, H.; Tian, S.; Guo, W.; Tu, X.; Zhao, J.; et al. Genome re-sequencing reveals the evolutionary history of peach fruit edibility. Nat. Commun. 2018, 9, 1-13. [CrossRef]

34. Meng, G.; Zhu, G.; Fang, W.; Chen, C.; Wang, X.; Wang, L.; Cao, K. Identification of loci for single/double flower trait by combining genome-wide association analysis and bulked segregant analysis in peach (Prunus persica). Plant Breed. 2019, 138, 360-367. [CrossRef]

35. Li, Y.; Cao, K.; Zhu, G.; Fang, W.; Chen, C.; Wang, X.; Zhao, P.; Guo, J.; Ding, T.; Guan, L.; et al. Genomic analyses of an extensive collection of wild and cultivated accessions provide new insights into peach breeding history. Genome Biol. 2019, 20,1-18. [CrossRef] [PubMed]

36. Guo, J.; Cao, K.; Deng, C.; Li, Y.; Zhu, G.; Fang, W.; Chen, C.; Wang, X.; Wu, J.; Guan, L.; et al. An integrated peach genome structural variation map uncovers genes associated with fruit traits. Genome Biol. 2020, 21, 1-19. [CrossRef] [PubMed]

37. Yadav, V.; Molina, I.; Ranathunge, K.; Castillo, I.Q.; Rothstein, S.J.; Reed, J.W. ABCG transporters are required for suberin and pollen wall extracellular barriers in Arabidopsis. Plant Cell 2014, 26, 3569-3588. [CrossRef] [PubMed] 
38. Zhao, G.; Shi, J.; Liang, W.; Zhang, D. ATP binding cassette G transporters and plant male reproduction. Plant Signal. Behav. 2016, 11,1-6. [CrossRef]

39. Wang, L.; Zhu, G. Descriptors and Data Standard for Peach (Prunus persica L.); China Agricultural Press: Beijing, China, 2005; pp. 21-22.

40. Verde, I.; Jenkins, J.; Dondini, L.; Micali, S.; Pagliarani, G.; Vendramin, E.; Paris, R.; Aramini, V.; Gazza, L.; Rossini, L.; et al. The Peach v2.0 release: High-resolution linkage mapping and deep resequencing improve chromosome-scale assembly and contiguity. BMC Genom. 2017, 18, 1-18. [CrossRef]

41. Li, H.; Durbin, R. Fast and accurate long-read alignment with Burrows-Wheeler transform. Bioinformatics 2010, 26, 589-595. [CrossRef]

42. McKenna, A.; Hanna, M.; Banks, E.; Sivachenko, A.; Cibulskis, K.; Kernytsky, A.; Garimella, K.; Altshuler, D.; Gabriel, S.; Daly, M.; et al. The genome analysis toolkit: A MapReduce framework for analyzing next-generation DNA sequencing data. Genome Res. 2010, 20, 1297-1303. [CrossRef]

43. Bradbury, P.J.; Zhang, Z.; Kroon, D.E.; Casstevens, T.M.; Ramdoss, Y.; Buckler, E.S. TASSEL: Software for association mapping of complex traits in diverse samples. Bioinformatics 2007, 23, 2633-2635. [CrossRef]

44. Turner, S.D. qqman: An R package for visualizing GWAS results using Q-Q and manhattan plots. J. Open Source Softw. 2018, 3, 731. [CrossRef]

45. Wang, K.; Li, J.; Wang, L.; Li, M.; Fang, Q. Study on characteristics of flower bud differentiation of different peach species. J. Fruit Sci. 2006, 23, 809-813. 